\title{
Gender similarity in low agreement between written and video ISAAC asthma questionnaires
}

\author{
M. Gharagozlou1, S. Khalili2, M. Hallajmofrad2, R. Mohammadzadeh2, \\ G. Mousavi2, H. Golkari3
}

ABSTRACT: Gender similarity in low agreement between written and video ISAAC asthma questionnaires. M. Gharagozlou, S. Khalili, M. Hallajmofrad, R. Mohammadzadeh, G. Mousavi, H. Golkari.

Background and Aim. The prevalence of childhood asthma varies among different nations and appears to have increased in recent years. It has been difficult to determine this prevalence precisely and compare the communities due to the lack of a standardized method. The International Studies of Asthma and Allergies in Childhood (ISAAC) has developed a written and video questionnaire to measure asthma prevalence and overcome these difficulties. This study was carried out to determine the prevalence of childhood asthma in Kashan, the central area of Iran, and to assess the agreement between the ISAAC written and video questionnaires and its gender relationship.

Methods. Between December 2002 and June 2003, 2533 school children, aged 13-14 years, in Kashan secondary schools completed sequentially the ISAAC written and video questionnaires. The agreement between responses to the two questionnaires for reported "ever wheeze", "exercise wheeze", "night wheeze" and "night cough" was determined, using kappa coefficient and concordance.

Results. The prevalence of asthma symptoms were significantly lower in the responses to the video questionnaire compared with the written questionnaire. Kappa coefficient showed only poor to fair agreement $(\kappa=0.13$ 0.24 ) between video and written questionnaires, although the concordance between them always exceeded $60 \%$.

Conclusions. This study showed that reported asthma symptoms based on video questionnaires were significantly lower than the written questionnaire, and that there is low agreement between the two questionnaires which requires further investigation to explain the findings. In addition, this investigation revealed no significant difference between the genders for this low agreement. Monaldi Arch Chest Dis 2006; 65: 4, 184-188.

Keywords: Asthma, ISAAC, prevalence, written questionnaires, video questionnaires.

${ }^{1}$ Immunology \& Allergy Dept., The Children Hospital Medical Center, Tehran University of Medical Sciences, Tehran,

2 Kashan University of Medical Sciences, Kashan,

3 Pediatrics Dept. Shaheed Beheshti University of Medical Sciences, Tehran, Iran.

Corresponding: Mohammad Gharagozlou; Dept of Immunology \& Allergy, The Children Hospital Medical Center, No 62, Dr Gharib St., Azadi Ave., 14194 Tehran, Iran; e-mail: gharagoz@Tums.ac.ir

\section{Introduction}

Childhood asthma is a major public health issue not only in Iran but also worldwide [1-4]. The prevalence of asthma in children varies among the nations and appears to have been increasing in recent years [5-8]. Comparison between the prevalence of asthma in different populations has not been easy due to the lack of a standardized method. Clinical examinations and tests including bronchial hyperresponsiveness have not been so practical for epidemiologic surveys, because of their high cost.

The International Study of Asthma and Allergies in Childhood (ISAAC) was set up to use systematic and standardized questionnaires to investigate asthma prevalence and its international comparisons [9]. The ISAAC written questionnaire was used in 56 countries up to 1998, and demonstrated important geographical variations in asth- ma prevalence [1]. Since the written questionnaire may result in problems of imprecise translation and vocabulary differences, an international version of the ISAAC video questionnaire has been developed (AVQ3.0) to overcome these difficulties and facilitate more reliable comparisons between populations $[10,11]$.

Various investigators have reported on a number of levels of agreement between the ISAAC written and video questionnaire (AVQ3.0) [12-21] and a few of them stratified the results according to gender without calculating the kappa coefficiency which is mandatory for this comparison [14, 15]. The aim of the present study was to compare these two questionnaires within a population of 13-14 year - old Persian - speaking Iranian children and show if there were differences among girls and boys. As with the other studies ISAAC provides a unique opportunity for us to investigate the prevalence of asthma symptoms and also to 
compare the audiovisual presentations with written forms. This survey also helps us to evaluate if the asthma symptoms interpretation is affected by the regional language.

\section{Methods}

This study was conducted in secondary schools in Kashan, the central area of Iran with tropical climate (fig. 1). The schools included in the study were randomly selected from all the schools in the area. School children aged 13-14 years were targeted for the investigation with a total number of 3,000. According to the ISAAC protocol, children aged 13-14 years were selected because they were able to self-complete the written and video questionnaires.

The response rate was $84.4 \%$, including 2533 children. The survey was carried out between December 2002 and June 2003. Written consent was obtained from the parents.

\section{Questionnaires}

The standardized ISAAC written questionnaire [9] and the ISAAC video questionnaire (AVA3.0) [10] were completed in the same session in school. The written questionnaire was translated into Persian following the ISAAC protocol by Iranian National Manager for ISAAC, regarding the ISAAC recommended

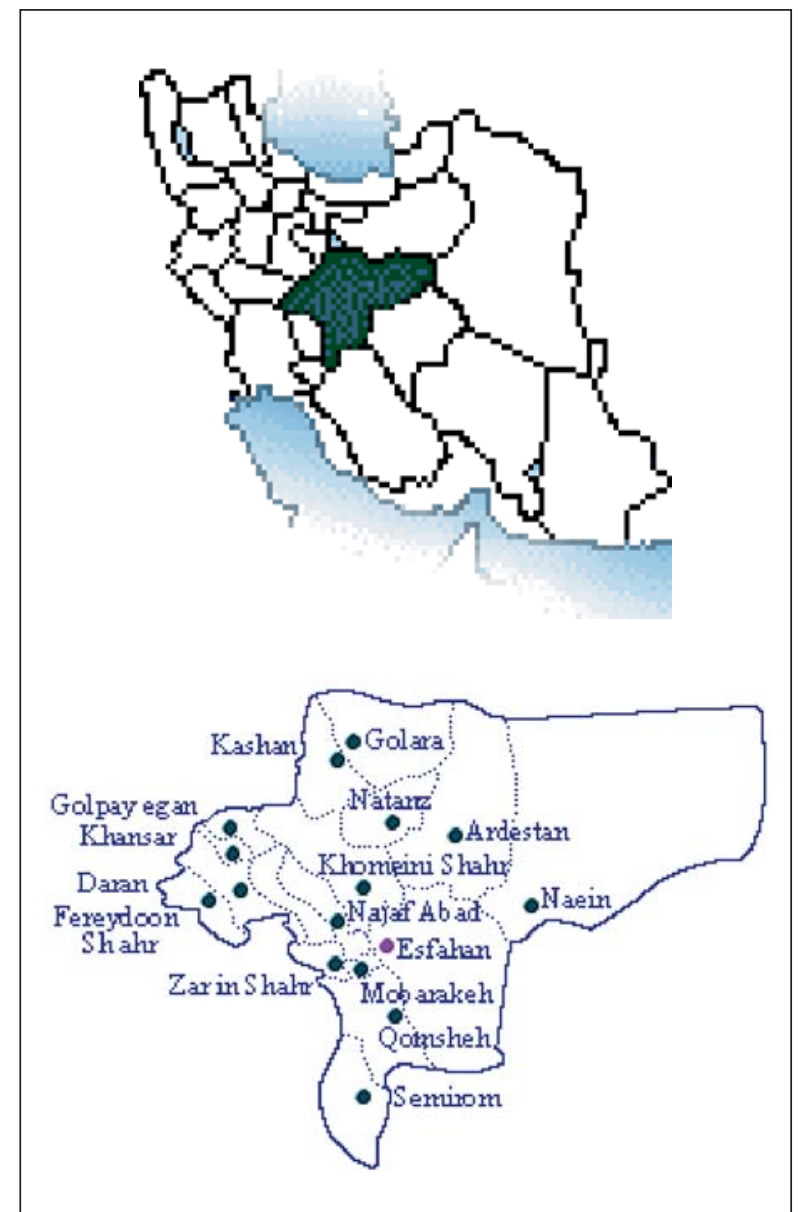

Fig. 1. - Map of Isfahan province and Kashan in Iran, the geographical area which our ISAAC study was carried out. back-translation. During the survey, written questionnaires were completed before video questionnaire to avoid potential order bias. The ISAAC written questionnaires asked for information on symptoms of wheezing and asthma. These questions included: 1) Have you ever had wheezing or whistling in the chest?

2) In the last 12 months, has your chest sounded wheezy during or after exercise?

3) In the last 12 months, has your sleep been disturbed due to wheezing?

4) In the last 12 months, have you had a dry cough at night, apart from a cough associated with a cold or chest infection?

The international video questionnaire (AVQ3.0) consisted of video sequences of young people of different races (African, Caucasian, Chinese and Indian) after exercise, waking at night wheezing, waking at night with a cough and severe asthma attack. After each sequence, the students were asked if they have ever experienced the same experiences as those shown in the video.

\section{Statistical analysis}

All data was entered using a computer software programme in accordance with ISAAC committee instructions. Data was analyzed using the statistical package for the social sciences (SPSS) software. The prevalence and $95 \%$ confidence intervals $(95 \% \mathrm{CI})$ were calculated for each symptom. A P- value of less than 0.05 was considered to be significant.

The agreement between video and written questionnaires was measured using the Kappa coefficient [23-25]. The strength of agreement by Kappa coefficient was appraised as recommended by Altman [11] $<0.20=$ poor, $0.21-0.40=$ fair, $0.41-0.60=$ moderate, $0.61-0.80=$ good, and $0.81-$ $1.0=$ very good. To compare the results with reports from other ISAAC studies, concordance was also calculated and Kappa coefficients were found from previously reported ISAAC investigations.

\section{Results}

Of the 3,000 Children, $2,533(84.4 \%)$ completed the written and video questionnaires with 1,100 (43.4\%) male subjects.

The prevalence of "ever wheeze", "exercise wheeze", "night wheeze" and "night cough" were $28.7 \%, 22.4 \%, 5.6 \%, 22.3 \%$ and $6.1 \%, 20.5 \%$, $3.2 \%, 20.4 \%$ in the written and video questionnaires, respectively (table 1). As the other previously ISAAC report about Iran [2], the doctor diagnosed asthma was reported significantly lower than asthma symptoms (table 1). This table shows that the prevalence of all symptoms in both written and video questionnaires were higher in the boys. The prevalence of symptoms were also higher in the written questionnaires rather than the video questionnaires.

Concordance between the two questionnaires was more than $60 \%$, whereas Kappa coefficient showed only poor to fair agreement for each question (table 2). The best agreement was for "exer- 
cise wheeze" and "night wheeze" questions that had Kappa values of 0.24 and 0.22 , respectively. Poor agreement was shown for "ever wheeze" and "night cough", with Kappa values of 0.13 and 0.15 , respectively. Although for the girls related Kappa coefficients were higher than for the boys, for each symptom, they were in the same or close range and none of them showed moderate or higher agreement. To compare our results with other reports of ISAAC studies, we collected the concordance and Kappa coefficient for agreement between written and video questionnaires using responses to "ever wheeze" question. Although there was a lack of Kappa values in the previous studies, these were calculated from their published data (table 3). In addition, the values were calculated for both genders separately to compare the results in each group (table 3 ).

Table 1. - Prevalence of asthma symptoms in the ISAAC written and video questionnaires in Kashan $n(\%)$

\begin{tabular}{lccccc}
\hline Questionnaire & Ever wheeze & Exercise wheeze & Night wheeze & Night cough & Doctor diagnosed asthma \\
\hline Written & & & & \\
Girls & $304(21.2)$ & $264(18.4)$ & $65(4.5)$ & $234(16.2)$ & $24(1.6)$ \\
Boys & $426(38.7)$ & $305(27.7)$ & $79(7.1)$ & $332(38.1)$ & $20(1.8)$ \\
Total & $728(28.7)$ & $569(22.4)$ & $144(5.6)$ & $566(22.3)$ & $44(1.7)$ \\
Video & & & & - \\
Girls & $76(5.3)$ & $252(17.5)$ & $34(2.3)$ & $254(17.7)$ & - \\
Boys & $80(7.2)$ & $264(24.4)$ & $48(4.3)$ & $265(24.0)$ & - \\
Total & $156(6.1)$ & $521(20.5)$ & $82(3.2)$ & $519(20.4)$ & \\
\hline
\end{tabular}

Table 2. - Agreement between ISAAC written and video questionnaire $\mathrm{n}(\%)$

\begin{tabular}{lccrrrr}
\hline $\begin{array}{l}\text { Written Questionnaire } \\
\text { Video questionnaire }\end{array}$ & $\begin{array}{c}\text { Yes } \\
\text { Yes }\end{array}$ & $\begin{array}{c}\text { Yes } \\
\text { No }\end{array}$ & $\begin{array}{c}\text { No } \\
\text { Yes }\end{array}$ & $\begin{array}{c}\text { No } \\
\text { No }\end{array}$ & Concordance & kappa \\
\hline $\begin{array}{l}\text { 1. Ever wheeze } \\
\quad \text { Girls }\end{array}$ & $47(3.2)$ & $257(17.9)$ & $29(2.0)$ & $1100(76.7)$ & $1147(0.8)$ & 0.17 \\
$\quad$ Boys & $47(4.2)$ & $377(34.2)$ & $33(3.0)$ & $643(58.4)$ & $690(0.62)$ & 0.07 \\
$\quad$ Total & $94(3.7)$ & $634(25.0)$ & $62(2.4)$ & $1743(68.8)$ & $1837(72.5)$ & 0.13 \\
2. Exercise wheeze & & & & & \\
$\quad$ Girls & $105(7.3)$ & $159(11.0)$ & $147(10.2)$ & $1022(71.3)$ & $1127(0.78)$ & 0.27 \\
$\quad$ Boys & $112(10.1)$ & $193(17.5)$ & $157(14.2)$ & $638(58.0)$ & $750(0.68)$ & 0.10 \\
$\quad$ Total & $217(8.5)$ & $352(13.8)$ & $304(12.0)$ & $1660(65.5)$ & $1877(74.1)$ & 0.24 \\
3. Night wheeze & & & & & \\
$\quad$ Girls & $14(0.9)$ & $51(3.5)$ & $20(1.3)$ & $1348(0.4)$ & $1362(0.95)$ & 0.26 \\
$\quad$ Boys & $13(1.1)$ & $66(6.0)$ & $35(3.1)$ & $986(89.6)$ & $999(0.90)$ & 0.15 \\
$\quad$ Total & $27(1.0)$ & $117(4.6)$ & $55(2.1)$ & $2334(92.1)$ & $2361(93.2)$ & 0.22 \\
4. Night cough & & & & & \\
$\quad$ Girls & $88(6.1)$ & $146(10.1)$ & $166(11.5)$ & $1033(72.0)$ & $1121(0.78)$ & 0.23 \\
$\quad$ Boys & $88(8.0)$ & $244(22.1)$ & $177(16.0)$ & $591(53.7)$ & $679(0.61)$ & 0.03 \\
$\quad$ Total & $176(6.9)$ & $390(15.3)$ & $343(13.5)$ & $1624(64.1)$ & $1800(71.6)$ & 0.15 \\
\hline
\end{tabular}

$\mathrm{P}<0.05$ considered as significant.

Table 3. - Reported agreement and concordance between the ISAAC video and written questionnaires for ever wheeze in different studies

\begin{tabular}{lclccc}
\hline Ever wheeze study & Ref. & Country & N & Concordance & Agreement kappa $^{\text {Agem }}$ \\
\hline Behbehani et al. & 20 & Kuwait & 3110 & 79 & 0.4 \\
Leung et al. & 15 & China & 4467 & 76 & 0.35 \\
Pizzichini et al. & 17 & Canada & & & 0.38 \\
& & Hamilton & 3051 & 71 & 0.37 \\
Lai et al. & 21 & Saskatoon & 1901 & - & 0.44 \\
Gibson et al. & 18 & China & 189 & 73 & 0.41 \\
Hong et al. & 19 & Korea & 157 & - & 0.45 \\
Gharagozlou et al. & This study & Iran & 2533 & 72 & 0.13 \\
\hline
\end{tabular}

${ }^{1}$ Kappa was calculated from published data. 


\section{Discussion}

Since there are difficulties relating to the definition of asthma and its epidemiological studies, the ISAAC questionnaires were developed. In particular the video questionnaire have facilitated comparisons between countries with different cultures and languages $[1,9,12]$. In our study, which has been carried out in the Persian - speaking Iranian children, it was revealed that the students find the video questionnaires far easier to understand and the questions are answered more without difficulty or need for explanation.

Several reported comparisons between these questionnaires have been carried out among school children from different cultural and language groups [12-20]. However none have been carried out with Persian - speaking children. Significant inconsistencies are seen between the two questionnaires probably because the video questionnaire is more objective and less affected by language and cultural factors [12]. On the other hand some authors found that language can affect the agreement between the two questionnaires and the lowest agreement is related to other languages like Russian [16]. However, it is not clear which questionnaire is the "gold standard" and provide more precise information about asthma.

The previous reported studies have revealed that the number of positive answers to the written questionnaire was higher than with the video questionnaire [12-22]. Our study has also shown similar results and positive responses were lower in the video questionnaire (table 1). This could be due to subjectivity of the written questionnaire which causes the students understand it differently and so overestimated prevalence of asthma may be shown. On the other hand, the scenes displayed on the video questionnaire demonstrate more severe feature forms of asthma especially in certain situation which some of the students have not experienced them. So the video questionnaire may underestimate the prevalence of asthma.

Our results regarding the comparison between the ISAAC written and video questionnaires are different from some other studies (table 2) $[12-15,20]$. Reported concordances in the other studies which are similar to ours, can not be the main determinant for high agreement between the two questionnaires, because they are heavily influenced by negative responses. Also concordance does not consider the proportion of agreement that occurred by chance, but kappa coefficient, which has been used in recent studies, is more precise and explains the agreement without the aforementioned defects [23-24]. The measured kappa in our study shows poor to fair agreement between written and video questionnaire. It means that there are discrepancies between the two questionnaires which are not related to culture and language.

Our data demonstrated no gender-specific differences in the questionnaires responses, by considering the kappa coefficients. Previous similar studies have not mentioned this aspect of the study.
In summary, we have shown that the agreement between the ISAAC written and video questionnaires in Iranian children is only poor to fair. Although it seems that the ISAAC video questionnaire can be an effective and valid tool for asthma epidemiological studies, further investigations are necessary to determine its precise properties.

Acknowledgements: The authors thank the school principals, teachers and children in Kashan for their cooperation, Kashan University of Medical Sciences Research Assistance and National Iranian ISAAC study group for their invaluable help

\section{References}

1. The International Study of Asthma and Allergies in Childhood (ISAAC) Steering Committee. Worldwide variation in prevalence of symptoms of asthma, allergic rhinoconjunctivitis, and atopic eczema: ISAAC. Lancet 1998; 351: 1225-132.

2. The International Study of Asthma and Allergies in Childhood (ISAAC) Steering Committee. Worldwide variations in the prevalence of asthma symptoms: the International Study of Asthma and Allergies in Childhood (ISAAC). Eur Respir J 1998; 12: 315-35.

3. Golshan M, Mohammad- Zadeh Z, Khanlar-Pour A, Iran -Pour R. Prevalence of asthma and related symptoms in junior high school children in Isfahan, Iran. Monaldi Arch Chest Dis 2002; 57: 19-24.

4. Golshan M, Mohammad - Zadeh Z, Zahedi - Nejad N, Rostam - Poor B. Prevalence of asthma and related symptoms in primary school children of Isfahan, Iran, in 1998. Asian Pac J Allergy Immunol 2001; 19: 163-7.

5. Lai CKW, Douglass C, Ho SS, et al. Asthma epidemiology in the far east. Clin Exp Allergy 1996; 26: 5-12.

6. Kim YY, CHO SH, Kim WK, et al. Prevalence of childhood asthma based on questionnaires and methacholine bronchial provocation test in Korea. Clin Exp Allergy 1997; 27: 761-8.

7. Bruney PJI, Chinn S, Rona RJ. Has the prevalence of asthma increased in children? Evidence from the national study of health and growth 1973 - 1986. BMJ 1990; 300: 1306-1310.

8. Cookson W, Moffatt M. Asthma: an epidemic in the absence of infection? Science 1997; 275: 41-42.

9. Asher MI, Keil U, Anderson HR, et al. International study of asthma and allergies in childhood (ISAAC): rationale and methods. Eur Respir J 1995; 8: 483-491.

10. Shaw R, Crane J, O'Donnell T, Lewis M, Srwart B, Beasley R. The use of videotaped questionnaire for studying asthma. prevalence. MJA 1992; 157: 311-4.

11. Shaw R, Crane J, Pearce N, et al. Comparison of a video questionnaire with the IUATLD written questionnaire for measuring asthma prevalence. Clin Exp Allergy 1992; 22: 561-8.

12. Shaw RA, Crane J, O'Donnell TV, Lewis ME, Stewart $\mathrm{B}$, Beasley R. The use of a videotaped questionnaire for studying asthma prevalence. A pilot study among New Zealand adolescents. Med J Aust 1992; 157: 311-314.

13. Pearce N, Weiland S, Kejl U, et al. R. Self - reported prevalence of asthma symptoms in children in Australia, England, Germany and New Zealand: an international comparison using the ISAAC protocol. Eur Respir J 1993; 1455-1461.

14. Pekkanen J, Remes ST, Husmann T, et al. Prevalence of asthma symptoms in video and written questionnaires among children in four regions of Finland land. Eur Respir J 1997; 10:1787-1794 
15. Leung R, Wong G, Lau J, et al. Prevalence of asthma and allergy in Hong Kong school children: an ISAAC study. Eur Respir J 1997; 10: 354-360.

16. Crane J, Mallol J, Beasley R, Stewart A, Asher MI; International Study of Asthma and Allergies in Childhood Phase 1 study group. Agreement between written and video questions for comparing asthma symptoms in ISAAC. Eur Respir J 2003; 21: 455-61.

17. Pizzichini MMM,Rennie D, Senthilselvan A, Teylor B, Habbick BF, Sears MR. Limited agreement between written and video asthma symptom questionnaires. $P e$ diatr Pulmonol 2000; 30: 307-312.

18. Gibson PG, Henry R, Shan S, et al. Validation of the ISAAC video questionnaire (AVQ3.0) in adolescents from a mixed ethnic background. Clin Exp Allergy 2000; 30: 1181-87.

19. Hong SJ, Kim SW, Oh JW, et al. The validity of the ISAAC written questionnaire and the ISAAC video questionnaire (AVQ 3.0) for predicting asthma associated with bronchial hyperreactivity in a group of 13-14 year old Korean schoolchildren. J Korean Med Sci 2003; $18: 48-52$
20. Behbehani NA, Abal A, Syabbalo NC, Azem AA. Prevalence of asthma, allergic rhinitis, and eczema in 13 - to 14- year - old children in Kuwait: An ISAAC study. Ann Allergy Asthma Immunol 2000; 85: 58-63.

21. Lai CKW. Chan JKW, Chan A, et al. Comparison of the ISAAC video questionnaire (AVQ 3.0) with the ISAAC written questionnaire for estimating asthma associated with bronchial hyperreactivity. Clin Exp Allergy 1997; 27: 540-545.

22. Hong SJ, Lee MS, Soha MJ, et al. Self - reported risk factors of asthma among Korean adolescents: 5 year follow up study, 1995 - 2000. Clin Exp Allergy 2004; 34: 1556-62.

23. Altman DG. Inter- rater agreement. In: Altman DG, editor. Practical statistics for medical research. London: Chapman \& Hall; 1991. p 403-405.

24. Streiner DL, Norman GR. Kappa Coefficient. In: Streiner DL, Norman GR, editors. Health measurement scales: a practical guide to their development and use. Oxford University Press; 1995. p 116-118.

25. Norman GR, Streiner DL. Cohen's kappa. In: Norman GR, Streiner DL, editors. Biostatistics. The bare essentials. ST. Louis: Mosby Year Book; 1994. p 164-168.

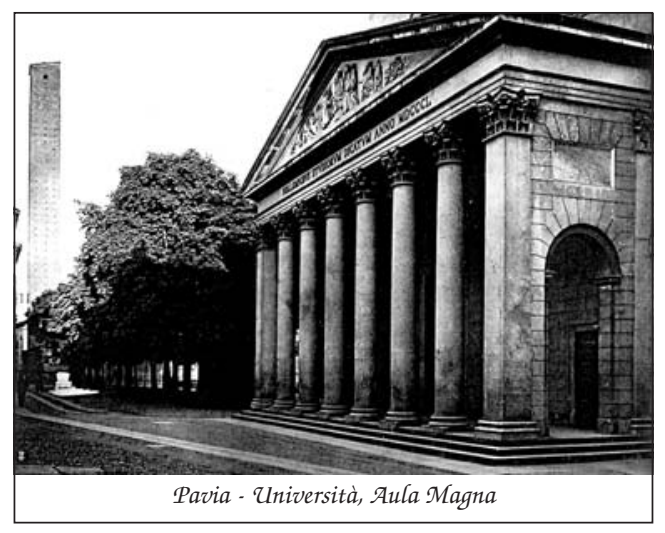

